\title{
PANORAMA DEL TECNICO DI DIALISI
}

\section{La redazione di A.N.T.E. news}

Approfittando dello spazio collaborativo gentilmente riservato da questo giomale all'A.N.T.E. , pubblichiamo un articolo che riteniamo possa aprire un confronto sui profili professionali utili all'applicazione di una corretta terapia dialitica. In particolare gradiremmo conoscere, attraverso queste pagine, il punto di vista di altri operatori sulla figura del TECNICO di DIALISI, figura che noi riteniamo essere, ogni giomo di più, indispensabile per un modemo trattamento dialitico.

\section{Infermiere $0 . .$. nurse? L'interazione con altri è utile allo scopo...}

A lle soglie del 2000 sarebbe opportuno cominciare a concepire l'assistenza ideale in dialisi attraverso una metamorfosi dell'attuale modello operativo e strutturale che ha influenzato e caratterizzato finora il personale ivi operante.

Per spiegarmi meglio vorrei partire da una analisi dell'epistemologia infermieristica, nella quale è definita la sistemazione del "sapere infermieristico" attraverso l'esame delle "strutture concettuali" con cui si costruisce il ragionare.

Essa considera, infatti, analizzandole ed esprimendole, le modalità con le quali le diverse discipline si articolano e si rapportano all'interno del complesso sapere assistenziale.

In essa traspare che l'Infermiere responsabile del Nursing è convinto che non vi è crescita, né personale né professionale, senza un adeguato percorso formativo e di aggiornamento.

Traspare altresì che nei confronti di altre aree disciplinari, l'Infermiere è, DEVE ESSERE, consapevole che non è sufficiente comprendere la necessità e l'importanza dell'INTERDISCIPLINARIETÀ ma che è necessario acquisire la relativa abilità a lavorare interagendo con altre figure, in modo autonomo e nel rispetto della professionalità degli altri operatori.

E consapevole, infatti, che da solo non riuscirà a realizzare il proprio obbiettivo di Nursing, cioè il massimo confort della persona con problemi di salute, in particolare nella direzione della ricerca e nei campi applicativi di specialità.

Per ritornare alla dialisi, la metamorfosi consisterà nel capire che la persona in trattamento dialitico ha come bisogno primario l'ELIMINAZIONE, a cui sono deputate apparecchiature tecnologicamente complesse, ma ad esso sono abbinati innumerevoli bisogni sia fisiologici che psicologici.

Dovrà allora capire che LUI (l'INFERMIERE) è sì deputato ad esaudire tutti i bisogni del paziente, ma dovrà per forza maggiore collaborare con altre figure - in particolar modo il Tecnico di dialisi con cui dovrà avere uno stretto rapporto di collaborazione se:

a) vuol essere Infermiere responsabile di Nursing;

b) vuole davvero che il paziente in dialisi raggiunga quella sicurezza e quel confort tanto auspicati.

Tutto ciò in considerazione del fatto che una diversa forma di metamorfosi snaturerebbe l'operato per cui l'infermiere è portato, ossia l'assistenza, essendo deviato verso altri compiti a cui può sì far fronte ma in maniera non sempre ottimale ritornando ancora una volta verso quella figura PARAMEDICA punto di partenza per il cambiamento.

Menegaldo Carmelo Infermiere professionale specializzato 